Editorial

\title{
Acknowledgment to Reviewers of Chemistry in 2020
}

\author{
Chemistry Editorial Office
}

Citation: Chemistry Editorial Office.

Acknowledgment to Reviewers of Chemistry in 2020. Chemistry 2021, 3, 146-148. https://doi.org/10.3390/ chemistry3010012

Published: 26 January 2021

Publisher's Note: MDPI stays neutral with regard to jurisdictional claims in published maps and institutional affiliations.

Copyright: (C) 2021 by the author. Licensee MDPI, Basel, Switzerland. This article is an open access article distributed under the terms and conditions of the Creative Commons Attribution (CC BY) license (http://creativecommons.org/licenses /by/4.0/).

MDPI AG, St. Alban-Anlage 66, 4052 Basel, Switzerland

Peer review is the driving force of journal development, and reviewers are gatekeepers who ensure that Chemistry maintains its standards for the high quality of its published papers. Thanks to the cooperation of our reviewers, in 2020, the median time to first decision was 14 days and the median time to publication was 33 days. The editors would like to express their sincere gratitude to the following reviewers for their precious time and dedication, regardless of whether the papers were finally published:

Achilias, Dimitris S.

Adly, Frady G.

Airapetian, Vladimir

Arunachalam, Prabhakarn

Barendt, Timothy

Bartoli, Mattia

Basarić, Nikola

Basile, Teodora

Bäßler, Ralph

Baumann, Marcus

Behrends, Volker

Berkefeld, Andreas

Bernasconi, Roberto

Bilewicz, Aleksander

Bilyachenko, Alexey N.

Bogomyakov, Artem

Bohinc, Klemen

Boratyński, Przemysłąw

Boris, Pejin

Bouquillon, Sandrine

Brack, André

Brittain, William D.G.

Calandra, Pietro

Calisi, Nicola

Canola, Sofia

Capaldo, Luca

Carda, Miguel

Carlucci, Lucia

Castro, Ricardo A.E.

Cavaliere, Chiara

Chang, Ching-yao

Chase, P. Bryant

Chastanet, Guillaume

Ciccone, Lidia

Colonna, Bianca
Connon, Stephen J.

Cortijo, Miguel

Crowley, James

Czapik, Agnieszka

Czugler, Mátyás

D’Acquarica, Ilaria

D'Urso, Alessandro

Da Silva, João Ferreira

Da Silva, José P.

Da Silva, Márcio José

Dalgarno, Scott

Dance, Ian

De La Torre, Maria Del Carmen

De Lázaro, Sérgio Ricardo

De Mendoza, Javier

De Sousa, Damião Pergentino

De Vleeschouwer, Freija

Deacon, Glen

Di Donato, Mariangela

Di Pietro, Sebastiano

Djuran, Miloš I.

Dobosz, Robert

Dolegowska, Barbara

Donaldson, Logan W.F.

Drabowicz, Jozef

Eremin, Dmitry

Escárcega Bobadilla, Martha Verónica

Fedin, Matvey

Ferdov, Stanislav

Fianchini, Mauro

Fiore, Michele

Forbes, Malcolm D. E.

Forrer, Daniel

Fraczyk, Justyna

Frampton, Chris 
Fromm, Katharina

Gaan, Sabyasachi

Gambi, Alberto

Gargiulo, Valentina

Garrido, Narciso M.

Gazizov, Almir

Genoni, Alessandro

Giannossa, Lorena Carla

Gładysz-Płaska, Agnieszka

Gobeze, Habtom B

González, Aridane G.

Graebin, Cedric

Grošelj, Uroš

Gross, Aaron

Gruzdev, Matvey

Guralsky, Illia

Gurzadyan, Gagik

Gütschow, Michael

Haas, Michael

Habata, Yoichi

Hadade, Niculina

Hahn, Dongyup

Hanton, Lyall

Hartshorn, Richard

He, Yingxin

Hecel-Czaplicka, Aleksandra

Hiraoka, Shuichi

Hiscock, Jennifer

Hoch, Constantin

Housecroft, Catherine

Hsu, Day-Shin

Huang, Xiaoming

Isab, Anvarhusein

Itabashi, Yutaka

Izadyar, Anahita

Jablan, Jasna

Jakić, Miće

Januszewski, Rafał

Jaros, Sabina W.

Jatkowska, Natalia

Jokić, Stela

Joó, Ferenc

Jurcek, Ondrej

Kabanos, Themistoklis

Karcz, Dariusz

Kawaguchi, Shin-ichi

King, Robert Bruce

Kobayashi, Kensei

Kodrin, Ivan

Kolodiazhnyi, Oleg Ivanov

Konieczny, Piotr
Korytowski, Witold

Koyioni, Maria

Krivoshein, Arcadius V.

Kubiak, Katarzyna

Kuppusamy, Senthil Kumar

Kusior, Anna

Łapok, Łukasz

Latajka, Zdzisław

Lee, Wonhye

Lemmerer, Andreas

Li, Feng

Li, Jiao Jiao

Liebing, Phil

Lihi, Norbert

Lima, Dênis

Lin, Shaoyang

Liu, Maning

Liu, Shiuh-Tzung

Lu, Mei-Chin

Lukšič, Miha

Lutz, Martin

Macetti, Giovanni

Mackereth, Cameron

Maeder, Marcel

Manzano, Blanca R.

Maranzana, Andrea

Martinez Gonzalez, Jose Angel

Martins, Luisa

Matera, Carlo

Matilla-Hernández, Antonio

Mayans, Julia

Mayer, Christian

Mayer-Gall, Thomas

Meille, Valerie

Meninno, Sara

Messi, Patrizia

Michalska-Domańska, Marta

Mijin, Dušan Ž.

Milcovich, Gesmi

Minami, Yasunori

Mketo, Nomvano

Monari, Antonio

Montiel-Smith, Sara

Morita, Yuji

Morozov, Vitaly A.

Mota, Antonio J.

Muñoz, Marcelo Andrés

Nalbandian, Michael J.

Nam, Ki Hyun

Nauroozi, Djawed

Nazarski, Ryszard B. 
Neut, Christel

Oliver, Allen G.

Osório, Wislei R

Padnya, Pavel

Palenzuela López, José Antonio

Pawar, Shrikant D

Pawlak, Tomasz

Pejchal , Jaroslav

Perera, Manomi

Pérez Sestelo, José

Petelenz, Piotr

Pimienta, Rodney Lacret

Piña Capó, Ma Nieves

Pizarro, Ana

Poirot, Marc

Porter, Ned

Prabakaran, Mayakrishnan

Prandi, Cristina

Pratesi, Alessandro

Putta, Anjaneyulu

Ragusa, Andrea

Rahman, Ashiqur X

Rahman, Md Toufiqur

Raithby, Paul

Ramasamy, Elamparuthi

Rapa, Mattia

Rezanka, Pavel

Ribeiro, Ana Paula Da Costa

Rice, Craig

Robl, Christian

Romański, Jarosław

Ronson, Tanya

Rosencrantz, Ruben R.

Saccoliti, Francesco

Saito, Shinichi

Salomon, Robert G.

San Fabian, Emilio

Santillán, Rosa

Sauer, Daniel

Savić, Aleksandar

Schäfer, André

Ścianowski, Jacek

Scrimin, Paolo

Seidel, Rüdiger W.

Serezhkin, Viktor N.

Setaka, Wataru

Shankhari, Pritam
Sharma, Ashutosh

Shiga, Takuya

Silva, Catarina L.

Silvente-Poirot, Sandrine

Silvestru, Cristian

Sinha, Abhijeet

Somsák, László

Sørby, Magnus Helgerud

Stanculescu, Ioana

Stefanelli, Manuela

Stefanowicz-Hajduk, Justyna

Stubbe, JoAnne

Sun, Qing-Fu

Sunatsuki, Yukinari

Suzuki, Nozomu

Szabelski, Paweł

Szafert, Slawomir

Takaichi, Shinichi

Takayanagi, Toshiyuki

Tan, Xiaoping

Tang, Jin-Kui

Tang, Longteng

Taniguchi, Tsuyoshi

Tao, Jun

Tava, Aldo

Thapa, Surendra

Tosato, Giovanna

Valkenier, Hennie

Vasu, Dhananjayan

Veríssimo, Marta

Ward, Michael D.

Wen, Jin

Weththimuni, Maduka Lankani

Wipf, David O.

Wnuk, Stanislaw

Wolny, Juliusz A.

Wrigstedt, Pauli

Wu, Li-Ming

Wu, Wangsuo

$\mathrm{Wu}$, Xin

Wzietek, Pawel

Wzorek, Alicja

$\mathrm{Xu}$, Minghao

Yamato, Takehiko

Zaltariov, Mirela-Fernanda

Zhang, Wei 